\title{
A Sensor System for the Monitoring of Production Processes of Low FODMAP Food ${ }^{+}$
}

\author{
Marco Santonico ${ }^{1}$, Simone Grasso ${ }^{1,}$, Giorgio Pennazza ${ }^{1}$, Luca Vollero ${ }^{2}$, Fabio Campoli ${ }^{3}$, \\ Alessandro Zompanti ${ }^{1}$, Giuseppe Ferri ${ }^{4}$ and Luca Piretta ${ }^{5}$ \\ 1 Unit of Electronics for Sensor Systems, Department of Engineering, Campus Bio-Medico University of \\ Rome, Via Alvaro del Portillo, 21-00128 Rome RM, Italy; m.santonico@unicampus.it (M.S.); \\ g.pennazza@unicampus.it (G.P.); a.zompanti@unicampus.it (A.Z.) \\ 2 Unit of Computational Systems and Bioinformatics, Department of Engineering, Campus Bio-Medico \\ University of Rome, Via Alvaro del Portillo, 21-00128 Rome RM, Italy; 1.vollero@unicampus.it \\ 3 Azioni Gastronomiche srl, Via della Tenuta del Cavaliere, 1-00012 Guidonia Montecelio RM, Italy; \\ campolifabio@gmail.com \\ 4 Department of Industrial and Information Engineering and Economics, University of L'Aquila, Via \\ Camponeschi, 19-67100 L'Aquila AQ, Italy; giuseppe.ferri@univaq.it \\ 5 Course of Food Science and Nutrition, Campus Bio-Medico University of Rome, Via Alvaro del Portillo, \\ 21-00128 Rome RM, Italy; pirets@tiscali.it \\ * Correspondence: s.grasso@unicampus.it; Tel.: +39-06-225-419-466 \\ + Presented at the Eurosensors 2018 Conference, Graz, Austria, 9-12 September 2018.
}

Published: 3 December 2018

\begin{abstract}
Irritable bowel syndrome is one of the most common gastrointestinal disorder. Despite its high prevalence, the factors responsible for the onset of the clinical symptoms are not clear yet. Recently, there was growing evidence the origin of IBS resides in a number of fermentable short-chain carbohydrates called FODMAPs. The present study assessed the possibility of using a multisensory system to detect the presence of FODMAPs in foods.
\end{abstract}

Keywords: FODMAP; sensor system; BIONOTE; irritable bowel syndrome

\section{Introduction}

Nowadays, the irritable bowel syndrome (IBS) is one of the most common gastrointestinal disorders reaching a prevalence of about $15 \%-20 \%$ in developed countries [1]. The IBS symptoms, which include abdominal cramping, bloating, constipation and/or diarrhea, can markedly interfere with patients' quality of life. While the causes of the pathology are not completely clear yet, there are evidences IBS etiology resembles the one of other well-known food intolerances. The consumption of specific food containing poorly absorbed short-chain carbohydrates by individuals affected by IBS is usually correlated with bacteria fermentation and osmotic fluid retention in the intestines [2]. The acronym FODMAPs has been coined to classify these types of malabsorbed carbohydrates, specifically identifiable as fermentable oligosaccharides, disaccharides, monosaccharides, and polyols [3]. Despite limited data, the restriction of FODMAPs in the diet has been identified as crucial tool in the therapeutic strategies of IBS [4-6]. Nonetheless, the set of molecules included in FODMAP group are difficult to measure both individually and collectively and at the moment no tools detecting their presence in foods are available. Moreover, the presence of these fermentable substances can vary tremendously depending on the way of cooking. In the present study, the feasibility of the innovative BIONOTE liquid sensor to analyze FODMAP molecules has been evaluated [7]. 


\section{Materials and Methods}

The voltammetric sensor employed in the liquid analyses consists of a sensing platform including a Screen-Printed Electrode (SPE; DRP-250BT, Metrohm, Herisau, Switzerland) probe (Working: Gold; Counter: Platinum; Reference: Silver), and a dedicated electronic interface providing the input signal and recording the output data. When the SPE is immersed in a solution, the applied input triangular waveform from $+1 \mathrm{~V}$ to $-1 \mathrm{~V}$, starts inducing oxi-reduction phenomena to the analytes dissolved in the aqueous media. The current generated by the electrons involved in such reactions is converted in voltage by a trans-impedance circuit. Some technical details: input signal frequency: $0.01 \mathrm{~Hz}$; output acquired with a rate of $200 \mathrm{~ms}$ (500 samples per measurement). All samples were poured into a disposable plastic cuvette and five independent analysis cycles were run.

The calibration of the voltammetric sensor against typical cereal's FODMAP was performed using inulin (I2255, Sigma Aldrich, St. Louis, MO, USA) and FOS (F8052, Sigma Aldrich) chemical standards, both dissolved in oligomineral water at the final concentration of $0.10,0.25$, $0.50,0.75,1.00,2.50,5.00,7.50,10.00 \mathrm{mg} / \mathrm{mL}$. To evaluate the influence of cooking temperature in the modification of the FODMAP electrochemical fingerprint, two independent solutions of inulin and FOS standards at the concentration of $10.00 \mathrm{mg} / \mathrm{mL}$ were brought to boil and aliquots were saved from the bulk solution every $60 \mathrm{~s}$ up to the 11th cooking minute. The solutions were left to cool at room temperature and then were analyzed with the voltammetric sensor. The calibration of the voltammetric sensor against typical legume's FODMAP was performed using the raffinose (I2255, Sigma Aldrich) chemical standard, dissolved in oligomineral water at the final concentration of $0.10,0.25,0.50,0.75,1.00,2.50,5.00,7.50,10.00 \mathrm{mg} / \mathrm{mL}$.

Commercial dry legumes requiring soaking step before their cooking were chosen to investigate FODMAP release. In two independent experiments, a quantity of $80 \mathrm{~g}$ of lentils and chickpeas were left soaked in $250 \mathrm{~mL}$ of oligomineral water at room temperature for $4.5 \mathrm{~h}$ and $10 \mathrm{~h}$, respectively. Aliquots were saved from the bulk solution every $30 \mathrm{~min}$ up to the end of the set soaking time. Samples were immediately analyzed by the voltammetric sensor without any modification following the procedure described above. To remove any residual soil trace, either legumes were washed twice in $250 \mathrm{~mL}$ of oligomineral water before the soaking. The reported data are an average of at least three independent experimental activities performed under the same conditions.

Differently from classic voltammetric data analysis where single current spikes are investigated, the approach used in this work made use of the whole electrochemical profile provided by the samples. To get a simplified representation of the recorded multidimensional data set that still highlighting the most important variables describing the investigated phenomena, multivariate data analysis techniques were applied. Principal Component Analysis (PCA) and Partial Least Square Discriminant Analysis (PLS-DA), were performed using PLSToolbox (Eigenvector Research Inc., Manson, WA, USA) in the Matlab Environment (The MathWorks, Natick, MA, USA).

\section{Results}

In the present study, the BIONOTE device has been employed to analyze liquids being in contact with food products during the cooking stages with the aim to capture information about FODMAPs content. As a preliminary approach to real sample analysis, the liquid sensor was challenged against FODMAP chemical standards with calibration purposes. From the widespread family of FODMAP compounds, inulin and FOS have been chosen as major representative of cereal derived product like pasta, while raffinose has been considered the most characteristic of legumes such as lentils and chickpeas. The PLS-DA models calculated on the calibration data set of each chemical standard solution have shown Root Mean Square Error in Cross Validation (RMSECV), using the Leave One Out criterion, of $1.99 \mathrm{mg} / \mathrm{mL}, 0.18 \mathrm{mg} / \mathrm{mL}$ and $1.29 \mathrm{mg} / \mathrm{mL}$ for inulin, FOS and raffinose, respectively (Figure 1a-c). Considering the calculated 
FODMAP release in the tested experimental setup is about $0.7-1 \mathrm{mg} / \mathrm{mL}$, the voltammetric sensor has shown to be able to predict inulin, FOS and raffinose concentration with acceptable resolution.
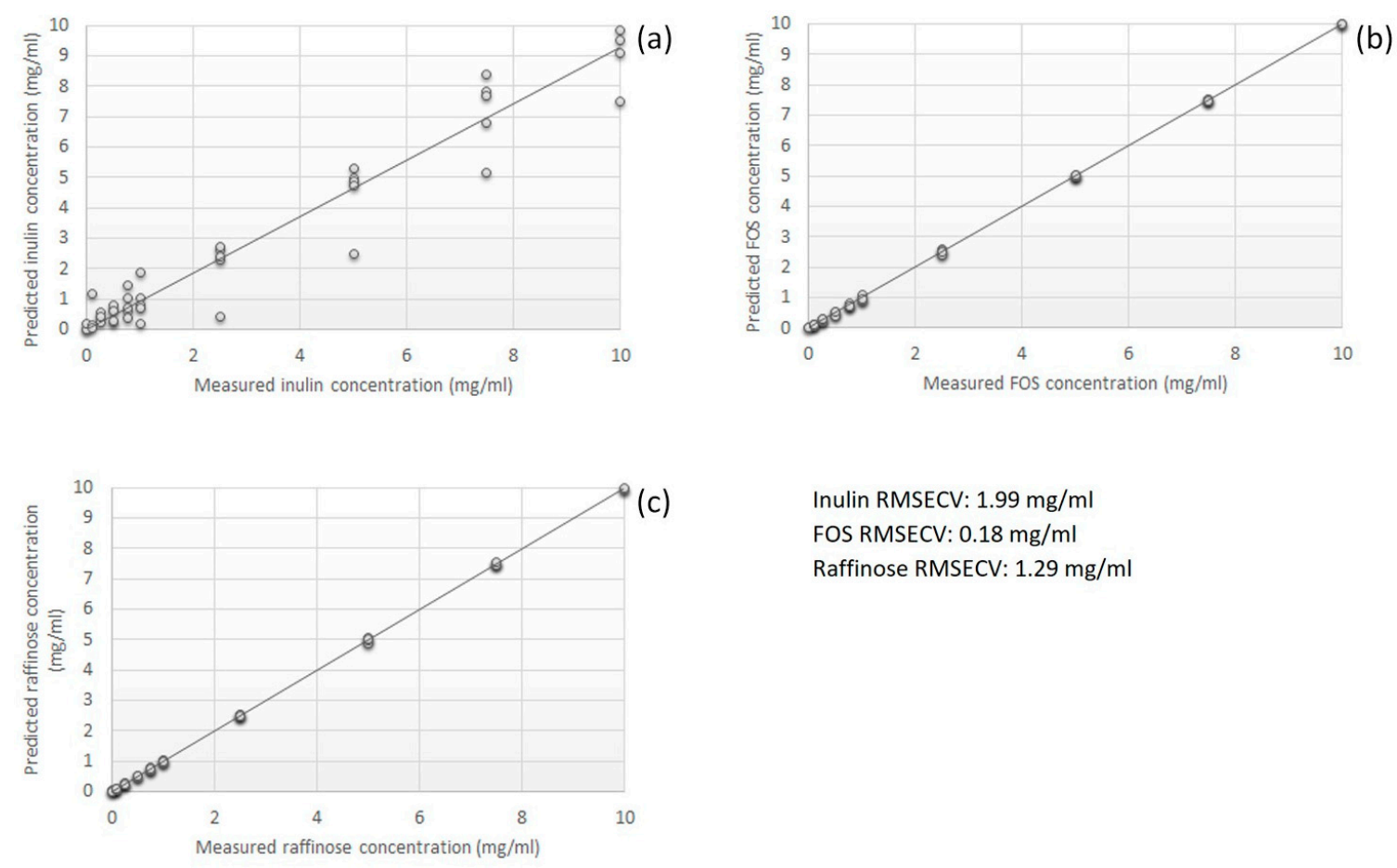

Figure 1. Calculated PLS-DA model for the prediction of (a) inulin, (b) FOS and (c) raffinose chemical standard liquid solutions.

Despite their salutary properties, legumes are considered one of the first food to avoid for the individuals affected by IBS due to their high content in FODMAP. For experimental purposes, lentils and chickpeas have been selected and samples from their aqueous media were collected and analyzed every $30 \mathrm{~min}$ for the entire duration of the soaking procedure. The comparison of the voltammetric fingerprints obtained from each collected sample has revealed a clear modification of the liquid media along with the soaking time for both the legumes (Figure 2).

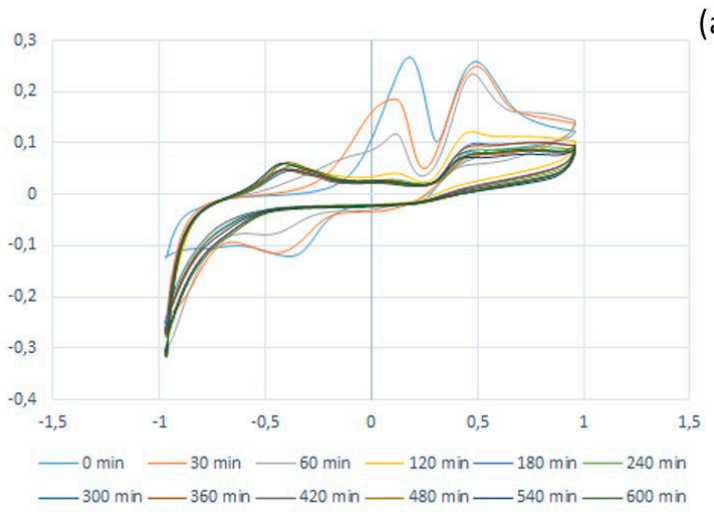

(a)

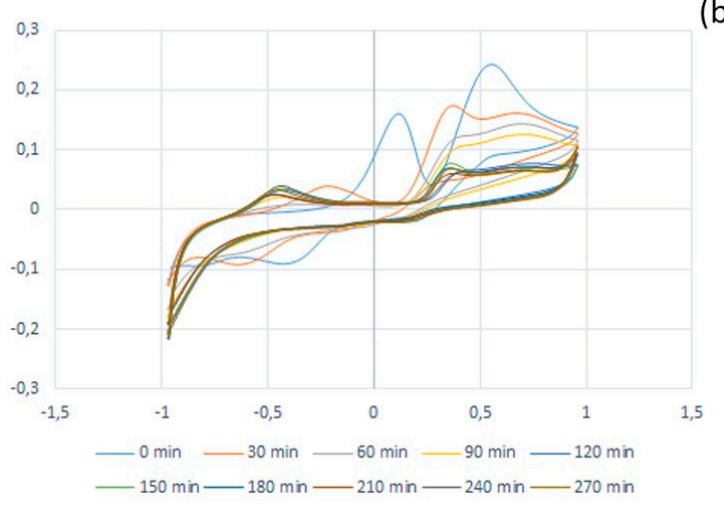

Figure 2. Electrochemical fingerprint of FODMAP related compounds released during (a) chickpeas and (b) lentils soaking for $10 \mathrm{~h}$ and $4.5 \mathrm{~h}$, respectively.

Moreover, the results of the two experimental activities were compared with the overall electrochemical fingerprint of the raffinose standard solutions in order to find differences and similarities. The score plot obtained from the calculated PCA model confirmed the time dependent trend observed in the electrochemical fingerprint and highlighted a nearly overlap of the FODMAP signal deriving from lentils and chickpeas. Besides the initial similarity, the 
progressive difference detected by the voltammetric sensor may be correlated with the complex mixture of compounds that were released by legumes compared to the single raffinose molecule and with their increasing concentration over the time.

\section{Discussion}

In the present work, the release of the FODMAP compounds by lentils and chickpeas during the soaking procedure has been investigated as a potential strategy to reduce their final content in the meal. Besides the legumes, pasta is one of the most representative food of the Mediterranean diet. However, while its benefic role is undoubted, recent researches are highlighting the involvement of this food as trigger of IBS in certain predisposed individuals due to its high content in FODMAP. BIONOTE platform has been already successfully applied in other food applications and the detection of FODMAP compounds during the cooking procedure of pasta will be the next challenge where the liquid sensor will be evaluated $[8,9]$. All this data together could play a decisive role in suggesting not only the type of food to be consumed by patients with IBS but also indicating how to prepare and cook them in a modulated diet.

Author Contributions: L.P., G.P. and M.S. conceived and designed the experiments; S.G., A.Z. and F.C. performed the experiments; M.S., L.V. and G.F. analyzed the data; S.G. and A.Z. contributed reagents/materials/analysis tools; L.P., S.G. and G.F. wrote the paper.

Conflicts of Interest: The authors declare no conflict of interest.

\section{References}

1. Hungin, A.P.S.; Whorwell, P.J.; Tack, J.; Mearin, F. The prevalence, patterns and impact of irritable bowel syndrome: An international survey of 40,000 subjects. Aliment. Pharmacol. Ther. 2003, 17, 643650, doi:10.1046/j.1365-2036.2003.01456.x.

2. Mertz, H.; Morgan, V.; Tanner, G.; Pickens, D.; Price, R.; Shyr, Y.; Kessler, R. Regional cerebral activation in irritable bowel syndrome and control subjects with painful and nonpainful rectal distention. Gastroenterology 2000, 118, 842-848, doi:10.1016/S0016-5085(00)70170-3.

3. Gibson, P.R.; Shepherd, S.J. Personal view: Food for thought-western lifestyle and susceptibility to Crohn's disease. The FODMAP hypothesis. Aliment. Pharmacol. Ther. 2005, 21, 1399-1409, doi:10.1111/j.1365-2036.2005.02506.x.

4. Piacentino, D.; Rossi, S.; Piretta, L.; Badiali, D.; Pallotta, N.; Corazziari, E. Tu1425 Role of FODMAPs, and Benefit of Low-FODMAP Diet, in Irritable Bowel Syndrome Severity. Gastroenterology 2016, 150, S901, doi:10.1016/S0016-5085(16)33048-7.

5. Piacentino, D.; Rossi, S.; Piretta, L.; Badiali, D.; Pallotta, N.; Corazziari, E.S. Low-FODMAP-diet in irritable bowel syndrome offers benefits not only in terms of gastrointestinal symptoms, but also in terms of psychopathology in the medium-and long-term. Eur. Psychiatry 2016, 33, S487, doi:10.1016/j.eurpsy.2016.01.1419.

6. Varjú, P.; Farkas, N.; Hegyi, P.; Garami, A.; Szabó, I.; Illés, A.; Solymár, M.; Vincze, Á.; Balaskó, M.; Bajor, J.; et al. Low fermentable oligosaccharides, disaccharides, monosaccharides and polyols (FODMAP) diet improves symptoms in adults suffering from irritable bowel syndrome (IBS) compared to standard IBS diet: A meta-analysis of clinical studies. PLoS ONE 2017, 12, e0182942, doi:10.1371/journal.pone.0182942.

7. Santonico, M.; Pennazza, G.; Grasso, S.; D'Amico, A.; Bizzarri, M. Design and test of a biosensor-based multisensorial system: A proof of concept study. Sensors 2013, 13, 16625-16640, doi:10.3390/s131216625. 
8. Santonico, M.; Grasso, S.; Genova, F.; Zompanti, A.; Parente, F.R.; Pennazza, G. Unmasking of olive oil adulteration via a multi-sensor platform. Sensors 2015, 15, 21660-21672, doi:10.3390/s150921660.

9. Santonico, M.; Parente, F.R.; Grasso, S.; Zompanti, A.; Ferri, G.; D’Amico, A.; Pennazza, G. Investigating a single sensor ability in the characterisation of drinkable water: A pilot study. Water Environ. J. 2016, 30, 253-260, doi:10.1111/wej.12186. article distributed under the terms and conditions of the Creative Commons Attribution (CC BY) license (http://creativecommons.org/licenses/by/4.0/). 